\title{
Niveis de interleucina-6 e fator de necrose tumoral-alfa no liquor de recém-nascidos a termo com encefalopatia hipóxico-isquêmica
}

\author{
Levels of interleukin-6 and tumor necrosis factor-alpha in the cerebrospinal fluid \\ of full-term newborns with hypoxic-ischemic encephalopathy
}

Rita de Cássia Silveira ${ }^{1}$, Renato S. Procianoy ${ }^{2}$

\section{Resumo}

Objetivo: avaliar os níveis liquóricos de IL-6 e TNF- $\alpha$ em recém-nascidos a termo com encefalopatia hipóxico-isquêmica(EHI), comparando-os com os de recém-nascidos controles.

Metodologia: estudo caso-controle realizado no período de julho de 1999 a outubro de 2001, incluindo dois grupos de recémnascidos a termo: controle, com 20 recém-nascidos sem sepse e/ou meningite e com escore de Apgar $\geq 9$ no primeiro e quinto minutos de vida; e casos, com 15 recém-nascidos asfixiados, caracterizados pelo escore de Apgar $\leq 4 \mathrm{e} \leq 6$ no primeiro e quinto minutos de vida, respectivamente, $\mathrm{pH}$ umbilical $<7,20 \mathrm{e} / \mathrm{ou}$ lactato arterial umbilical $>3,0 \mathrm{mmol} / \mathrm{l}$ e necessidade de ventilação com pressão positiva pelo menos durante 2 minutos após o nascimento. Foram coletadas amostras de liquor nas primeiras 48 horas de vida, para determinação dos níveis de IL-6 e TNF- $\alpha$ pelo método de enzimoimunoensaio.

Resultados: os grupos não diferiram quanto ao peso de nascimento, idade gestacional, classificação quanto ao peso e idade gestacional, tipo de parto e tempo médio de obtenção do liquor; seus exames foram obtidos em média com 17 horas de vida. Nos recémnascidos asfixiados, as medianas dos níveis liquóricos foram: 157,5 $\mathrm{pg} / \mathrm{ml}$ para IL-6 e $14,7 \mathrm{pg} / \mathrm{ml}$ para TNF- $\alpha$, significativamente mais elevadas que nos controles (IL-6: 4,1 pg/ml e TNF- $\alpha$ : 0,16 pg/ml).

Conclusões: recém-nascidos a termo com EHI apresentaram níveis liquóricos de IL-6 e TNF- $\alpha$ mais elevados que controles, possivelmente devido à produção local cerebral dessas citocinas, especialmente o TNF- $\alpha$. Estes achados estimulam estudos futuros, utilizando bloqueadores cerebrais das ações dessas citocinas como estratégia de neuroproteção.

J Pediatr (Rio J) 2003;79(4):297-302: encefalopatia hipóxicoisquêmica, asfixia perinatal, interleucina-6, fator de necrose tumoral-alfa, citocinas.

\begin{abstract}
Objective: to determine cerebrospinal fluid levels of interleukin6 and tumor necrosis factor-alpha in full-term infants with hypoxicischemic encephalopathy, comparing with control infants.

Methods: controlled, prospective study, performed between July 1999 and October 2001 with two groups of full-term newborns: 20 controls with no sepsis and/or meningitis and Apgar score $\geq 9$ at first and fifth minutes; and cases, 15 asphyxiated full-term newborns with Apgar $\leq 4$ and $\leq 6$ at first and fifth minutes, umbilical blood cord $\mathrm{pH}<7.20$ and/or umbilical arterial blood lactate $>3.0 \mathrm{mmol} / \mathrm{L}$, and requiring positive pressure ventilation for at least 2 minutes after birth. Cerebrospinal fluid samples were collected within 48 hours of birth for determination of interleukin- 6 and tumor necrosis factoralpha by enzyme immunoassay.
\end{abstract}

Results: groups were similar concerning birthweight, gestational age, type of delivery and mean time required for cerebrospinal fluid sample collection. The samples were collected at mean with 17 hours of life. The medians cerebrospinal fluid levels in asphyxiated newborn infants were: $157.5 \mathrm{pg} / \mathrm{ml}$ for interleukin- 6 and $14.7 \mathrm{pg} / \mathrm{ml}$ for tumor necrosis factor-alpha, significantly higher than the controls (interleukin-6: $4.1 \mathrm{pg} / \mathrm{ml}$ and tumor necrosis factor-alpha: $0.16 \mathrm{pg} / \mathrm{ml}$ ).

Conclusions: full-term newborns with hypoxic-ischemic encephalopathy present higher cerebrospinal fluid interleukin- 6 and tumor necrosis factor-alpha levels than the controls, possibly because of the local cerebral production of these cytokines, especially tumor necrosis factor-alpha. These results support a recommendation for future studies with brain blockers of the actions of these cytokines for neuroprotective strategies.

J Pediatr (Rio J) 2003;79(4):297-302: hypoxic-ischemic encephalopathy, perinatal asphyxia, interleukin-6, tumor necrosis factor-alpha, cytokines.

1. Doutora em Pediatria - Universidade Federal do Rio Grande do Sul, médica Neonatologista do Hospital de Clínicas de Porto Alegre (HCPA).

2. Professor Titular de Pediatria - Universidade Federal do Rio Grande do Sul, Chefe do Serviço de Neonatologia do HCPA, pesquisador 1 A CNPq.

Fontes finaciadoras: CNPq e FIPE-HCPA.

Artigo submetido em 12.02.03, aceito em 31.03.03. 


\section{Introdução}

Apesar dos recentes avanços na monitorização biofísica e bioquímica do feto no trabalho de parto e no parto, a asfixia perinatal ainda contribui intensamente na morbidade e mortalidade neonatal, ocorrendo em dois a cinco recém-nascidos para cada 1.000 nascimentos vivos ${ }^{1,2}$. É o resultado de diversas condições que podem interferir com a troca de gases feto-placentária, levando à hipoxemia progressiva, hipercapnia e acidose metabólica; como conseqüência clínica da lesão hipóxico-isquêmica no cérebro do recém-nascido, desenvolve-se a encefalopatia hipóxicoisquêmica (EHI), manifestação clínica da asfixia perinatal mais descrita e conhecida ${ }^{3,4}$. Os mecanismos fisiopatológicos da EHI têm sido objeto de interesse recente, com a finalidade principal de desenvolver estratégias de neuroproteção a partir do conhecimento bioquímico e celularhumoral das lesões cerebrais.

Diversas evidências indicam o envolvimento da cascata inflamatória na patogênese da lesão isquêmica cerebral. A reação inflamatória, acionada pela isquemia no sistema nervoso central (SNC), caracteriza-se pelo influxo de leucócitos, incluindo polimorfonucleares seguidos pelos monócitos, e pela ativação da microglia. Para tanto, é necessário a expressão de moléculas de adesão específicas, fatores quimiotáticos e citocinas pró-inflamatórias. As citocinas são proteínas de baixo peso molecular, produzidas e secretadas pelos monócitos, macrófagos, células endoteliais e fibroblastos e, no SNC, são produzidas e secretadas pela microglia e pelos astrócitos ${ }^{5-7}$.

Modelos experimentais sugerem o envolvimento de diversas citocinas, principalmente interleucina-6 (IL-6) e fator de necrose tumoral- $\alpha$ (TNF- $\alpha$ ) no dano isquêmico cerebral $^{8-12}$. O TNF- $\alpha$ e a interleucina-1ß (IL-1ß) encontram-se elevados após isquemia cerebral, podendo induzir uma reação inflamatória no SNC, juntamente com a IL-6. Além disso, parecem modular diretamente o processo de apoptose das células no SNC, promovendo diferenciação, proliferação e subseqüente infiltração leucocitária ${ }^{13,14}$. Em adultos, tem sido demonstrada a correlação direta entre os níveis de IL-6 e TNF- $\alpha$ no liquor e o prognóstico neurológico após isquemia cerebral aguda ${ }^{15}$.

Em recém-nascidos a termo com encefalopatia hipóxico-isquêmica (EHI), os níveis liquóricos de TNF- $\alpha$ e de IL$1 \beta$ foram mais elevados naqueles com alterações neurológicas aos 12 meses de idade ${ }^{16}$. Martín-Ancel e colaboradores encontraram níveis de IL-6 no liquor de recém-nascidos com manifestações neurológicas graves significativamente mais elevados que nos com encefalopatia moderada ou leve, no entanto estes autores não avaliaram a concentração do TNF- $\alpha$ liquórico ${ }^{17}$. A maioria dos estudos que investigaram os mecanismos de lesão cerebral após asfixia perinatal são experimentais, sendo necessário estudos clínicos de recém-nascidos com EHI avaliando o comportamento da IL-6 e do TNF- $\alpha$ no SNC, uma vez que se acredita que essas duas citocinas sejam as mais identificadas com o processo inflamatório de hipóxia-isquemia no período neonatal.
A proposta deste estudo foi avaliar se a concentração de IL-6 e de TNF- $\alpha$ no liquor é mais elevada em recémnascidos a termo com EHI, comparando-os com recémnascidos normais (controles).

\section{Pacientes e métodos}

Foi realizado um estudo caso-controle, incluindo todos os recém-nascidos a termo nascidos no centro obstétrico do Hospital de Clínicas de Porto Alegre e admitidos na UTI neonatal, com diagnóstico de asfixia perinatal ou com necessidade de realização de punção lombar nas primeiras 48 horas de vida, para investigação de sepse neonatal precoce, durante o período de julho de 1999 a outubro de 2001.

O diagnóstico de asfixia perinatal foi dado pela presença de no mínimo três critérios dentre os referidos abaixo; sendo obrigatória a presença dos critérios 3 ou $4^{1,18,19}$ :

1) sinais de sofrimento fetal documentado pela monitorização intraparto (MAP), como desacelerações persistentes, bradicardia fetal sustentada ou "MAP silente";

2) escores de Apgar inferior ou igual a 4 no primeiro minuto e inferior ou igual a 6 no quinto minuto;

3) valor de $\mathrm{pH}$ umbilical inferior a 7,20;

4) lactato de sangue arterial umbilical superior a $3,0 \mathrm{mmol} / 1$;

5) necessidade de ventilação, com pressão positiva no mínimo durante dois minutos, para iniciar esforço respiratório. O diagnóstico de encefalopatia hipóxicoisquêmica foi estabelecido pela presença de asfixia perinatal associada a manifestações neurológicas decorrentes da hipoxemia e isquemia ${ }^{16}$. EHI foi classificada de acordo com os critérios de Sarnat e Sarnat ${ }^{20}$.

O grupo controle foi constituído de recém-nascidos não-asfixiados, com escore de Apgar $\geq 9$ no primeiro e quinto minutos de vida, e que tiveram necessidade de exame de liquor como parte da investigação para sepse neonatal, sendo a antibioticoterapia, nestes casos, suspensa em menos de 48 horas, com boa evolução clínica. Este grupo teve hemograma e hemocultura coletados pelo médico-assistente antes de iniciar antibioticoterapia, com resultado normal, e coletaram-se exames laboratoriais, devido a uma suspeita inicial de sepse neonatal precoce.

Foram excluídos os recém-nascidos com suspeita clínica ou laboratorial de infecção congênita, presença de sepse e/ou meningite, malformação congênita, acidentes de punção lombar durante realização de exame do liquor, recémnascidos com crises convulsivas sem relação com evento hipóxico e de etiologia não esclarecida, drogadição materna, mães com qualquer infecção do grupo STORCH durante a gestação, ou com soropositividade para o vírus da imunodeficiência humana (HIV+), utilização materna de opiáceos ou drogas depressoras respiratórias no período do periparto.

Os recém-nascidos do grupo controle e os casos foram acompanhados durante a internação até o momento da alta 
hospitalar, e nos casos foram realizados acompanhamento neuropediátrico, eletroencefalograma e ultra-sonografia cerebral transfontanelar.

A partir de uma amostra solicitada pela equipe assistencial, como parte da investigação rotineira do paciente, foi obtido um volume adicional de liquor, não implicando em coleta exclusivamente para a pesquisa. $\mathrm{O}$ liquor foi obtido pela punção lombar (500 $\mathrm{ml}$ de volume total) e em seguida congelado a $-70^{\circ} \mathrm{C}$. As amostras foram coletadas nas primeiras 48 horas de vida do recém-nascido, para dosagem posterior de IL-6 e TNF- $\alpha$. Todas as amostras foram testadas em duplicata. A técnica empregada para as dosagens de IL-6 e TNF- $\alpha$ foi enzimoimunoensaio (Quantikine Human IL-6 e TNF- $\alpha$, R\&D Systems, Inc. MN, USA). O limite de detecção para IL- 6 foi 0,7 pg/ml e para TNF- $\alpha 0,1$ $\mathrm{pg} / \mathrm{ml}$, com coeficientes de variação intra e interensaios para IL-6 e TNF- $\alpha$ inferiores a 5\%. A leitura foi realizada no leitor óptico automático Spectramax no comprimento de onda de $570 \mathrm{~nm}$.

\section{Análise estatística}

O tamanho da amostra foi calculado para um poder de 0,97 e significância de 0,05 . Os resultados foram expressos em média e desvio padrão ou mediana e variação. Foram empregados teste de qui-quadrado na análise das variáveis categóricas, teste $t$ de Student na comparação de dados paramétricos e teste de Mann-Whitney na comparação entre os níveis liquóricos de ambos os grupos. O nível de significância mínima aceito foi $\mathrm{p}<0,05$.

O protocolo do estudo foi aprovado pelo Comitê de Ética da nossa instituição e em todos os casos foi obtido consentimento informado do responsável.

\section{Resultados}

Foram incluídos no estudo trinta e cinco recém-nascidos $(n=35)$, sendo quinze casos e vinte controles. Os casos e controles não diferiram quanto ao peso de nascimento, idade gestacional, classificação quanto ao peso e idade gestacional, tipo de parto e tempo médio de obtenção do liquor. Não houve diferença significativa entre os grupos com relação ao tempo de vida dos recém-nascidos, quando da realização da punção lombar para obtenção do liquor. Os dois grupos tiveram seus exames obtidos em média com 17 horas de vida, e as medianas das horas de vida dos recémnascidos foram 8 (2-25) nos asfixiados com EHI e 10 (3-26) nos controles. Os escores de Apgar foram significativamente mais baixos nos recém-nascidos asfixiados (Tabela 1).

A coleta do liquor no grupo controle deveu-se às seguintes situações: infecção urinária materna não tratada ou tratamento incompleto em 4 recém-nascidos; ruptura prematura das membranas ovulares (tempo superior a 24 horas) em 8 recém-nascidos; febre no recém-nascido em 5 destes; e desconforto respiratório, com diagnóstico de taquipnéia transitória na evolução clínica, em 3 recémnascidos.
Tabela 1 - Características da população estudada

\begin{tabular}{lll}
\hline Grupo & EHI & Controle \\
\hline $\mathrm{N}$ & 15 & 20 \\
Peso de nascimento (g) & $3.439 \pm 320$ & $3.046 \pm 118$ \\
Idade gestacional (semanas) & $39,6 \pm 0,9$ & $38,1 \pm 0,4$ \\
Apgar 1 minuto* & $1(0-4)$ & $9(9-10)$ \\
Apgar 5 minuto* & $5(0-6)$ & $9(9-10)$ \\
Parto vaginal & $12(80 \%)$ & $14(70 \%)$ \\
AIG & $11(73 \%)$ & $14(70 \%)$ \\
Momento da coleta & $8(2-25)$ & $10(3-26)$ \\
(horas de vida) & & \\
\hline Dados expressos em média e desvio padrão ou mediana e variação. \\
*p<0,0001. \\
AlG: adequado para idade gestacional.
\end{tabular}

Os recém-nascidos asfixiados foram classificados em três estágios da EHI, conforme critérios de Sarnat e Sarnat; encontrando EHI grau I em 8 recém-nascidos (53\%), EHI grau II em 3 (20\%), e EHI grau III em 4 recém-nascidos (27\%). A ultra-sonografia cerebral foi realizada em todos os recém-nascidos asfixiados, evidenciado-se alteração em cinco casos (33\%), e edema cerebral difuso e hiperecogenicidade talâmica foram as alterações mais freqüentes. Dos 14 pacientes que fizeram eletroencefalograma, seis apresentaram alterações significativas atribuíveis à EHI (43\%), e o exame neurológico encontrava-se alterado na alta hospitalar ou momentos anteriores ao óbito em seis recémnascidos. Ocorreram três óbitos, todos recém-nascidos com EHI grave. Os dados avaliados exclusivamente nos recémnascidos com EHI encontram-se sumarizados na Tabela 2.

Os níveis de IL-6 no liquor foram significativamente mais elevados nos recém-nascidos asfixiados, quando comparados com recém-nascidos controles. Com relação ao TNF- $\alpha$, a diferença nos níveis liquóricos foi ainda mais evidente, com concentrações significativamente mais elevadas nos recém-nascidos asfixiados e praticamente indetectáveis no liquor de recém-nascidos controles (Tabela 3).

\section{Discussão}

As citocinas têm sido implicadas em diversos mecanismos, que podem potencializar a lesão isquêmica cerebral, tais como a liberação da forma induzível da enzima óxido nítrico sintetase pelos astrócitos; o recrutamento, a ativação e a adesão leucocitárias ao endotélio; a promoção do estado pró-coagulante endotelial e a regulação do processo da apoptose. A interleucina-6 e o TNF- $\alpha$ são citocinas que participam ativamente dos mecanismos de lesão cerebral que ocorrem na asfixia perinatal ${ }^{16,17,21}$. Os resultados do nosso estudo demonstram esses achados em recém-nascidos humanos com EHI e permitem comparar as suas concentrações liquóricas com as de recém-nascidos controles. As citocinas, principalmente a IL-6, afetam a diferenciação, o crescimento e a sobrevida in vitro das células neuronais, 
Tabela 2 - Características dos recém-nascidos asfixiados

\begin{tabular}{|c|c|c|c|c|c|c|c|}
\hline Paciente & Ultra-som & EEG* & Exame $^{\dagger}$ & EHI * & $\begin{array}{c}\text { pH } \\
\text { cordão umbilical }\end{array}$ & $\begin{array}{c}\text { Lac }(\mathbf{m m o l} / \mathrm{L}) \\
\text { cordão umbilical }\end{array}$ & $\begin{array}{c}\text { Ocorrência } \\
\text { de óbito }\end{array}$ \\
\hline 1 & $\mathrm{~N}$ & A & $\mathrm{N}$ & 2 & 6,78 & & Não \\
\hline 2 & $\mathrm{~N}$ & $\mathrm{~N}$ & $\mathrm{~N}$ & 1 & 6,99 & 13,9 & Não \\
\hline 3 & $\mathrm{~N}$ & & $\mathrm{~N}$ & 1 & 7,04 & 4,18 & Não \\
\hline 4 & A & A & A & 3 & 7,08 & 4,61 & Sim \\
\hline 5 & $\mathrm{~N}$ & $\mathrm{~N}$ & A & 2 & 6,73 & 4,09 & Não \\
\hline 6 & A & $\mathrm{N}$ & $\mathrm{N}$ & 1 & 6,95 & 4,50 & Não \\
\hline 7 & $\mathrm{~N}$ & $\mathrm{~N}$ & $\mathrm{~N}$ & 1 & 7,05 & 4,81 & Não \\
\hline 8 & $\mathrm{~N}$ & $\mathrm{~N}$ & $\mathrm{~N}$ & 1 & 6,87 & 4,80 & Não \\
\hline 9 & A & A & A & 3 & & 8,17 & Sim \\
\hline 10 & $\mathrm{~N}$ & $\mathrm{~N}$ & $\mathrm{~N}$ & 1 & & 3,87 & Não \\
\hline 11 & $\mathrm{~N}$ & A & A & 2 & 6,83 & 5,39 & Não \\
\hline 12 & $\mathrm{~N}$ & $\mathrm{~N}$ & $\mathrm{~N}$ & 1 & 7,02 & 4,60 & Não \\
\hline 13 & $\mathrm{~N}$ & $\mathrm{~N}$ & $\mathrm{~N}$ & 1 & 6,87 & 8,41 & Não \\
\hline 14 & A & A & A & 3 & 6,64 & 7,47 & Não \\
\hline 15 & A & A & A & 3 & 7,01 & 9,02 & Sim \\
\hline
\end{tabular}

*Eletroencefalograma, † Exame neurológico na alta/óbito, ‡ Grau de encefalopatia hipóxico-isquêmica, $\mathrm{A}=$ alterado, $\mathrm{N}=$ normal.

e em conseqüência, o cérebro do recém-nascido torna-se particularmente suscetível a alterações nas concentrações de citocinas ${ }^{22}$.

As nossas amostras foram obtidas em média com 17 horas de vida, fato de extrema relevância para o estudo porque a meia-vida das citocinas no plasma é muito curta, são produzidas e secretadas em resposta a diversos estímulos, com um pico de nível sérico muito precoce, já a meiavida das citocinas no liquor é pouco conhecida, mas acredita-se que apresente comportamento semelhante ${ }^{5,6} . \mathrm{Em}$ adultos com acidente vascular isquêmico, foram encontrados níveis de IL-6 mais elevados no liquor que no sangue nos primeiros 2 a 3 dias após o insulto, com o tempo estes níveis se equilibraram, sugerindo produção intratecal precoce de IL-6 ${ }^{23}$. A meia-vida da IL-6 no recém-nascido asfixiado é muito menor que no adulto com lesão isquêmica cerebral, provavelmente em função de diferenças nos está-

Tabela 3 - Níveis liquóricos de IL-6 e TNF- $\alpha$

\begin{tabular}{lccc}
\hline Grupo & EHI & Controle & p \\
\hline N & 15 & 20 & \\
IL-6 (pg/ml) & $157,5(66,8-288)$ & $4,1(2,5-5,8)$ & $<0,00001$ \\
TNF- $\alpha(\mathrm{pg} / \mathrm{ml})$ & $14,7(13,8-15,3)$ & $0,16(0-0,25)$ & $<0,000001$
\end{tabular}

Dados expressos em mediana e variação. Teste Mann-Whitney. gios de maturação do sistema imune ${ }^{17}$. O TNF- $\alpha$, por sua vez, começa a elevar-se na primeira hora após a oclusão da artéria cerebral média, com pico de resposta à isquemia em 6 a 12 horas $^{24,25}$. Portanto, a preocupação de coletarmos as amostras de liquor o mais precocemente possível foi fundamental para a adequada análise dos resultados.

Nossos resultados demonstraram níveis elevados de IL6 no liquor de recém-nascidos que sofreram asfixia perinatal, quando comparados com recém-nascidos controles. Tivemos o cuidado de excluir do grupo controle qualquer situação patológica que pudesse propiciar reação inflamatória com conseqüente elevação de citocinas no SNC. A origem exata da IL-6 na asfixia perinatal ainda permanece a definir, também não está claro onde a IL-6 participa, na degeneração ou no reparo dos neurônios após asfixia perinatal. Astrócito e microglia são possíveis fontes de IL-6 no $\mathrm{SNC}^{26}$. Os monócitos e neutrófilos, recrutados na isquemia cerebral, são capazes de produzir IL-6 em resposta ao estímulo do TNF- $\alpha$ e da IL-1 $1 \beta^{5,27}$. O que explica também a elevação muito significativa do TNF- $\alpha$ no liquor dos nossos recém-nascidos asfixiados.

Os recém-nascidos sujeitos à hipóxia-isquemia transitória, durante um episódio de asfixia ao nascimento, podem parecer relativamente normais logo após a reanimação na sala de parto, e apresentar evidências de lesão cerebral retardada algumas horas mais tarde, como as convulsões que ocorrem, caracteristicamente, nas primeiras 24 horas de vida ${ }^{28,29}$. Esse mecanismo de lesão cerebral retardado 
ainda não foi esclarecido, mas nas necropsias cerebrais de recém-nascidos que morreram por asfixia perinatal, foram encontradas células apoptóticas. É provável que o processo bifásico da fisiopatologia da asfixia perinatal esteja muito relacionado com os mecanismos de apoptose ${ }^{30}$. O TNF- $\alpha$ pode ser potente indutor da apoptose através da ativação da esfingomielinase, proporcionando um aumento das concentrações citosólicas de ceramida, que é potencializadora da apoptose ${ }^{30}$. Oygür e colaboradores avaliaram o valor preditivo dos níveis plasmáticos e liquóricos do TNF- $\alpha$ e da IL-1ß no prognóstico de recém-nascidos a termo com EHI e ambos são bons preditores de seqüelas precoces. As concentrações do TNF- $\alpha$ no liquor logo após o insulto hipóxico foram muito elevadas nos recém-nascidos que morreram, reforçando o papel do TNF- $\alpha$ como responsável pelo processo bifásico da lesão cerebral e pelo prognóstico desfavorável, devido à sua ação indutora da apoptose em células neuronais ${ }^{16}$.

A associação entre a gravidade e freqüência de óbitos com os níveis liquóricos de IL-6 e TNF- $\alpha$ não foi observada porque somente três recém-nascidos evoluíram para óbito, não permitindo análise estatística. No entanto, todos óbitos apresentaram EHI grau III e níveis mais elevados desses marcadores, demonstrando uma tendência possível de ser demonstrada com um número maior de casos. $\mathrm{O}$ cálculo do tamanho da amostra foi realizado para analisar a diferença nos níveis liquóricos de IL-6 e TNF- $\alpha$ entre asfixiados e controles, e não para estabelecer essa associação.

Interleucina- 6 e TNF- $\alpha$ parecem representar um papel importante na cascata de eventos inflamatórios da asfixia perinatal. Recém-nascidos a termo com EHI apresentaram níveis mais elevados de IL- 6 e TNF- $\alpha$ no liquor, atribuíveis a uma ação direta do estímulo asfíxico sobre o sistema nervoso central. A produção local cerebral do TNF- $\alpha$ e da IL-6 nos recém-nascidos que sofreram insulto hipóxicoisquêmico é provável, possibilitando estudos futuros com a finalidade de instituir novas modalidades terapêuticas, como bloqueadores cerebrais das ações dessas citocinas. Acreditamos, portanto, que este estudo contribuirá para sugerir o desenvolvimento de novas estratégias de neuroproteção em recém-nascidos com encefalopatia hipóxico-isquêmica.

\section{Agradecimentos}

Os autores agradecem aos professores e doutores da UFRGS: Célia Carlini e Mário Wagner, e à médica neonatologista, Clarissa Miura.

\section{Referências bibliográficas}

1. Chou YH, Tsou Yau KI, Wang PJ. Clinical application of the measurement of cord plasma lactate and pyruvate in the assessment of high-risk neonates. Acta Paediatr 1998;87:764-8.
2. Mulligan JC, Painter MJ, O'Donoghue PA, MacDonald HM, Allan AC, Taylor PM. Neonatal asphyxia. II. Neonatal mortality and long-term sequelae. J Pediatr 1980;96:903-7.

3. Lupton BA, Hill A, Roland EH, Whitfield MF, Flodmark O. Brain swelling in the asphyxiated term newborn: pathogenesis and outcome. Pediatrics 1988;82:139-46.

4. Williams CE, Mallard C, Tan W, Gluckman PD. Pathophysiology of perinatal asphyxia. Clin Perinatol 1993;20(2):305-25.

5. Clark WM. Cytokines and reperfusion injury. Neurology 1997;49(5 Suppl 4):10-4.

6. Cohen MC, Cohen S. Cytokine function: a study in biologic diversity. Am J Clin Pathol 1996;105:589-98.

7. Hirano T. The biology of interleukin-6. Chem Immunol 1992;51:153-80.

8. Szaflarski J, Burtrum D, Silverstein FS. Cerebral hypoxiaischemia stimulates cytokine gene expression in perinatal rats. Stroke 1995;26:1093-100.

9. Hagberg H, Gilland E, Bona E, Hanson LA, Hahin-Zoric M, Blennow M. Enhanced expression of Interleukin (IL)-1 and IL6 messenger RNA and bioactive protein after hypoxia-ischemia in neonatal rats. Pediatr Res 1996;40:603-9.

10. Stroemer RP, Rothwell NJ. Exacerbation of the ischemic brain damage by localized striatal injection of interleukin- $1 \mathrm{~b}$ in the rat. J Cereb Blood Flow Metab 1998;18:833-9.

11. Loddick SA, Turnbull AV, Rothwell NJ. Cerebral Interleukin-6 is neuroprotective during permanent focal cerebral ischemia in the rat. J Cereb Blood Flow Metab 1998;18:176-9.

12. Dihne M, Block F. Focal ischemia induces transient expression of IL-6 in the substantia nigra pars reticulata. Brain Res 2001;889(1-2):165-73.

13. Pulera MR, Adams LM, Liu H, Santos DG, Nishimura RN, Yang $\mathrm{F}$, et al. Apoptosis in a neonatal rat model of cerebral hypoxiaischemia. Stroke 1998;29:2622-30.

14. Tarkowski E, Rosengren L, Blomstrand C, Jensen C, Ekholm S, Tarkowski A. Intrathecal expression of proteins regulating apoptosis in acute stroke. Stroke 1999;30:321-7.

15. Vila N, Castillo J, Dávalos A, Chamorro A. Proinflammatory cytokines and early neurological worsening in ischemic stroke. Stroke 2000;31:2325-9.

16. Oygür N, Sönmez O, Saka O, Yegin O. Predictive value of plasma and cerebrospinal fluid tumor necrosis factor-a and interleukin- $1 \mathrm{~b}$ concentrations on outcome of full term infants with hypoxic-ischaemic encephalopathy. Arch Dis Child Fetal Neonatal Ed 1998;79:190-3.

17. Martín-Ancel A, García-Alix A, Pascual-Salcedo D, Cabanãs F, Valcarce M, Quero J. Interleukin-6 in the cerebrospinal fluid after perinatal asphyxia is related to early and late neurological manifestations. Pediatrics 1997;100:789-94.

18. Carter BS, Haverkamp AD, Merenstein GB. The definition of acute perinatal asphyxia. Clin Perinatol 1993;20:287-98.

19. Shirey T, Pierre JS, Winkelman J. Cord lactate, $\mathrm{pH}$, and blood gases from healthy neonates. Gynecol Obstet Invest 1996;41:15-9.

20. Sarnat HB, Sarnat MS. Neonatal encephalopathy following fetal distress: a clinical and electroencephalographic study. Arch Neurol 1976;33:696.

21. Shalak LF, Laptook AR, Jafri HS, Ramilo O, Perlman JM. Clinical chorioamnionitis, elevated cytokines, and brain injury in term infants. Pediatrics 2002;110:673-80.

22. Dammann O, Leviton A .Brain damage in preterm newborns: biological response modification as a strategy to reduce disabilities. J Pediatr 2000;136:433-8.

23. Tarkowski E, Rosengren L, Blomstrand C, Wikkelsö C, Jensen C, Ekholm S, et al. Early intrathecal production of interleukin-6 predicts the size of brain lesion in stroke. Stroke 1995;26:1393-8. 
24. Barone FC, Arvin B, White RF, Miller A, Webb CL, Willette $\mathrm{RN}$, et al. Tumor necrosis factor-a - a mediator of focal ischemic brain injury. Stroke 1997;28:1233-44.

25. Yamasaki Y, Matsuura N, Shozuhara H, Onodera H, Itoyama Y, Kogure K. Interleukin-1 as a pathogenetic mediator of ischemic brain damage in rats. Stroke 1995;26:676-81.

26. Maeda Y, Matsumoto M, Hori O, Kuwabara K, Ogawa S, Yan $\mathrm{SD}$, et al. Hypoxia/reoxygenation-mediated induction of astrocyte interleukin 6: a paracrine mechanism potentially enhancing neuron survival. J Exp Med 1994;180:2297-308

27. Silverstein FS, Barks JD, Hagan P, Liu XH, Ivacko J, Szaflarski J. Cytokines and perinatal brain injury. Neurochem Int 1997;30: 375-83.

28. Rivkin MJ, Volpe JJ. Hypoxic-ischemic brain injury in the newborn. Semin Neurol 1993;13:30-9.
29. Ahn OM, Korst LM, Phelan JP, Martin GI. Does the onset of neonatal seizures correlate with the timing of fetal neurologic injury? Clin Pediatr (Phila) 1998;37:673-6.

30. Mehmet H, Edwards AD. Hypoxia, ischaemia, and apoptosis. Arch Dis Child Fetal Neonatal Ed 1996;75:F73-5.

Endereço para correspondência:

Dra. Rita de Cássia Silveira

Rua General João Telles, 542/ 601

CEP 90035-121 - Porto Alegre, RS

E-mail: rita.c.s@terra.com.br 